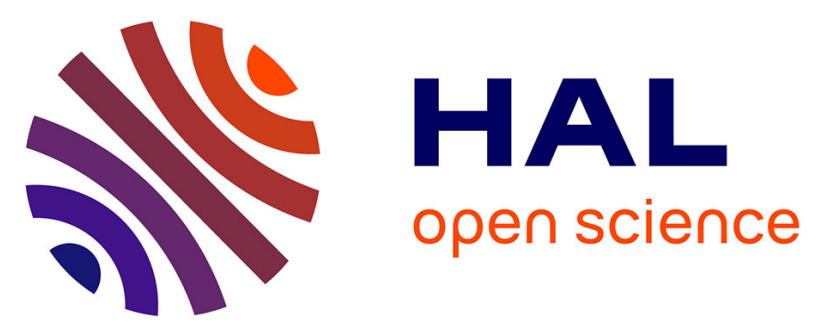

\title{
Influence of finite-size and edge effects on the exchange-bias properties of ferromagnetic/antiferromagnetic nanodots: Granular Monte Carlo investigation
}

\author{
Haydar Kanso, Renaud Patte, Vincent Baltz, Denis Ledue
}

\section{To cite this version:}

Haydar Kanso, Renaud Patte, Vincent Baltz, Denis Ledue. Influence of finite-size and edge effects on the exchange-bias properties of ferromagnetic/antiferromagnetic nanodots: Granular Monte Carlo investigation. Physical Review B: Condensed Matter and Materials Physics (1998-2015), 2019, 99, pp.054410. 10.1103/PhysRevB.99.054410 . hal-02015777

\section{HAL Id: hal-02015777 \\ https://hal.science/hal-02015777}

Submitted on 19 May 2019

HAL is a multi-disciplinary open access archive for the deposit and dissemination of scientific research documents, whether they are published or not. The documents may come from teaching and research institutions in France or abroad, or from public or private research centers.
L'archive ouverte pluridisciplinaire HAL, est destinée au dépôt et à la diffusion de documents scientifiques de niveau recherche, publiés ou non, émanant des établissements d'enseignement et de recherche français ou étrangers, des laboratoires publics ou privés. 


\title{
Influence of finite-size and edge effects on the exchange-bias properties of ferromagnetic/antiferromagnetic nanodots: Granular Monte Carlo investigation
}

\author{
Haydar Kanso, ${ }^{1, *}$ Renaud Patte, ${ }^{1}$ Vincent Baltz, ${ }^{2}$ and Denis Ledue ${ }^{1, \dagger}$ \\ ${ }^{1}$ Normandie Université, UNIROUEN, INSA Rouen, CNRS, GPM, F-76800 Saint Étienne du Rouvray, France \\ ${ }^{2}$ Université Grenoble Alpes, CNRS, CEA, Grenoble INP, INAC-Spintec, F-38000 Grenoble, France
}

(Received 5 October 2018; revised manuscript received 14 December 2018; published 11 February 2019)

\begin{abstract}
In this paper, we investigate exchange-biased square nanodots whose lateral sizes range between 130 and $500 \mathrm{~nm}$, in comparison with continuous films by kinetic Monte Carlo simulations. We use a granular model which takes into account disordered interfacial phases by considering less stable magnetic grains at the interface in the antiferromagnetic (AF) layer. We further model the effect of the nanofabrication process by considering grains with reduced surfaces at the edges, due to grain cutting. Since less stable grains at the nanodot edges in the AF layer have been experimentally evidenced, we assumed a weaker anisotropy for the grains which are in the AF layer at the dot edges. Our results evidence two different mechanisms of the ferromagnetic $(\mathrm{F})$ layer reversal depending on the magnitude of the coupling between $\mathrm{F}$ grains. In the weak coupling regime relative to the anisotropy, the exchange field is independent of the coupling and no variability from one nanodot to another is observed. By contrast, in the strong coupling regime, the exchange field depends on the coupling and it shows a high variability from one nanodot to another. Our model also well explain some experimental features observed in $\mathrm{NiFe} / \mathrm{IrMn}$ nanodots (for various lateral sizes) and continuous films, at various measurement temperatures and various AF thicknesses. Finally, our model explains a long lasting issue about why the exchange field in nanodots can be either smaller or larger than in continuous films.
\end{abstract}

DOI: 10.1103/PhysRevB.99.054410

\section{INTRODUCTION}

Exchange-bias (EB) effect occurs due to the exchange coupling at the interface between $\mathrm{F}$ and AF materials [1-4], leading to a shift of the hysteresis loop which is known as the EB field $\left(H_{E}\right)$. EB, mainly in F/AF bilayers, has been extensively investigated in the last few decades, from both experimental and theoretical points of view. In terms of technological applications, exchange-biased bilayers constitute an essential part of spin valves and magnetic tunnel junctions used in the field of spintronics [5,6]. More recently, the challenge becomes increasing the magnetic storage density and the miniaturization of devices (magnetic sensors, high-density data storage media) [7]. For that, it is crucial to extend the investigations of the EB mechanism to nanostructures [8]. In the case of exchange-biased bilayers, it is of particular interest to study how EB properties are modified when passing from a continuous F/AF film to nanodot arrays (with a lateral size of a few hundred nanometers). Indeed, EB properties depend on various parameters such as bulk anisotropies, bulk and interfacial exchange couplings, and grain volumes in polycrystalline films. Such a phenomenon becomes more complicated at the nanoscale, due to edge and finite-size effects which well affect EB properties compared to continuous films. Actually, contradictory results on $H_{E}$ have been reported. For example, in $\mathrm{NiFe} / \mathrm{IrMn}$ bilayers, it was found that $H_{E}$ at room temperature is smaller in nanodots for thicknesses $t_{\mathrm{IrMn}}<11 \mathrm{~nm}$

\footnotetext{
*haydar.kanso@etu.univ-rouen.fr

†denis.ledue@univ-rouen.fr
}

and larger above this thickness $[9,10]$. In a more recent investigation on $\mathrm{NiFe} / \mathrm{IrMn}$ nanodots $\left(t_{\mathrm{IrMn}} 7-8 \mathrm{~nm}\right)$ [11], it was observed that $H_{E}$, at room temperature, is smaller when the size decreases, while at $10 \mathrm{~K}$ it is larger with decreasing the size. Also, in $\mathrm{NiFe} / \mathrm{FeMn}$ square dots $\left(t_{\mathrm{FeMn}}=10 \mathrm{~nm}\right)$, it was shown that $H_{E}$ at room temperature decreases with the size decrease [12] in agreement with Refs. [9-11]. In contrast, it was observed that $H_{E}$ at room temperature is smaller in $\mathrm{NiFe} / \mathrm{IrMn}$ nanodots with $t_{\mathrm{IrMn}}=15 \mathrm{~nm}$ [13] in contradiction with Refs. [9-11]. In another recent study on $\mathrm{Co} / \mathrm{IrMn}$ square nanodots [14], it was reported that the dot lateral size has no significant effect on $H_{E}$ at room temperature $\left(3 \mathrm{~nm}<t_{\mathrm{IrMn}}<15 \mathrm{~nm}\right)$. It should be noted that $H_{E}$ versus $t_{\mathrm{IrMn}}$ exhibits a maximum at $t_{\mathrm{IrMn}}=6.5 \mathrm{~nm}$ for all dot sizes. In $\mathrm{Co} / \mathrm{CoO}$ nanostructures, for small Co thickness $\left(t_{\mathrm{Co}}=\right.$ $8 \mathrm{~nm}$ ) a strong increase in $H_{E}$ at $4 \mathrm{~K}$ was detected as the lateral size decreases [15]. However, at large Co thickness $\left(t_{\mathrm{Co}}=25 \mathrm{~nm}\right)$, the increase of $H_{E}$ is less pronounced. One possible explanation of the difference between nanodots and continuous films is the dot edges which induce additional locations for the formation of spin-glass-like AF regions [16]. So, according to the data previously mentioned, it seems that $H_{E}$ measured at room temperature decreases as the dot size decreases (at least for small AF thicknesses) while it is the opposite at low temperatures. However, these results depend on several conditions such as the AF layer thickness, the initial annealing temperature, and the lateral size of bilayers.

At the same time, there are only a few numerical studies of EB properties in F/AF nanodots. They are divided into two categories: those based on an atomistic model and others based on a granular one. An atomistic model assuming a 
small fraction of spins pinned ferromagnetically in the $\mathrm{AF}$ interface plane has been developed [17]. This study, based on Monte Carlo simulations, showed an increase of $H_{E}$ of patterned systems compared to a continuous film. Concerning the granular approach, a significant difference in $H_{E}$ between nanodots and continuous films has been reported [18]. However, such a granular model is rather simple since it does not take into account disordered interfacial phases (spin-glasslike regions) in the AF layer which are usually considered $[11,19,20]$. These interfacial phases can significantly affect the EB properties. In particular, at working temperatures for devices, they contribute to device-to-device variability of EB once the film is nanofabricated [21].

In this study, we investigate EB properties of square F/AF nanodots using a granular model which includes less stable magnetic regions at the F/AF interface [22,23]. In addition, due to the nanofabrication process, less stable grains at the nanodot edges in the AF layer are considered as experimentally demonstrated [16]. We first investigate the effect of the coupling between $\mathrm{F}$ grains on the variability of $H_{E}$ from one nanodot to another and on the $\mathrm{F}$ layer magnetization reversal mechanism. Then we study EB properties at room temperature of nanodots in comparison with continuous films for various AF thicknesses. Moreover, we investigate the nanodot size effects on the temperature dependence of $H_{E}$. In these two cases, our results are successfully compared to recent experimental data on $\mathrm{NiFe} / \mathrm{IrMn}$ bilayers. Our investigations are performed using kinetic Monte Carlo simulations.

The remainder of the paper is organized as follows. The model and simulation technique are described in Sec. II. Numerical results and discussions are given in Sec. III. A conclusion is given in Sec. IV.

\section{MODEL AND SIMULATION}

In order to simulate EB properties of F/AF bilayers, we generate a system of two layers with a $\mathrm{F}$ layer of thickness $t_{\mathrm{F}}$ and an AF layer of thickness $t_{\mathrm{AF}}$. Since columnar growth is assumed, the two layers have the same granular microstructure which is generated by Voronoi tessellation in two dimensions [24]. To model disordered interfacial phases [11,19,20], small grains (SG) of thickness $t_{\mathrm{SG}}<t_{\mathrm{AF}}$ are randomly distributed over the F/AF interface within the AF layer [22,23]. Such disordered interfacial phases are produced by magnetic frustration which is induced by the defects at the F/AF interface (e.g., interlayer diffusion and stacking faults). So, we assume that these SG exhibit altered magnetic properties compared to those of the AF bulk. It worth noting that a difference between nanodots and continuous films is the small number of grains (few hundreds) in nanodots due to their reduced lateral sizes. In addition, to be close to real nanodots, we model the effect of the nanofabrication process by the presence of grains with reduced surfaces at the edges due to grain cutting [Fig. 1(a)]. Moreover, we assume that those grains which are located in the $\mathrm{AF}$ layer have the same altered magnetic properties as the SG [Fig. 1(b)] [16]. To ease the discussion, these grains located at the edges in the $\mathrm{AF}$ layer will be referred as $\mathrm{SG}_{\mathrm{E}}$. It should be noted that $t_{\mathrm{SG}}=t_{\mathrm{AF}}$ is larger than $t_{\mathrm{SG}}$ [see Fig. 1(b)]. Consequently, the blocking temperature $T_{\mathrm{B}}$ distribution within a given nanodot might differ from that of a (a)
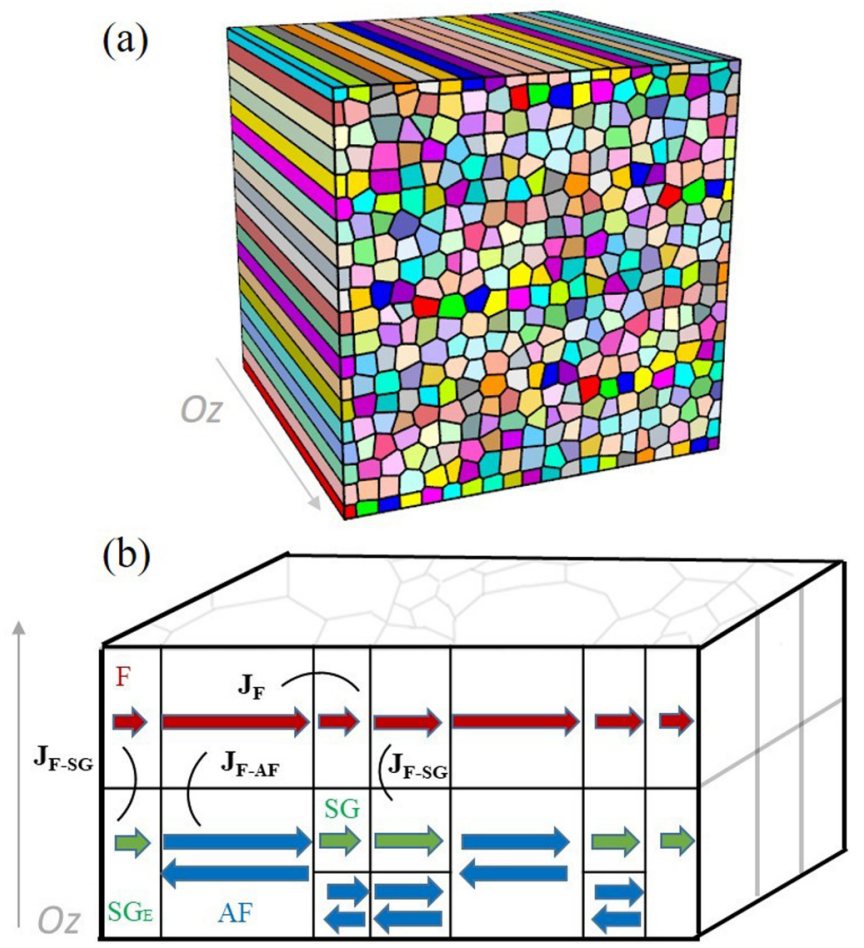

FIG. 1. (a) Top view of the granular microstructure of a nanodot with grains of reduced surfaces at the edges. (b) Sketch of the F/AF nanodot with SG randomly spread over the F/AF interface in the AF layer and $\mathrm{SG}_{\mathrm{E}}$ at the edges in the $\mathrm{AF}$ layer (in green).

continuous film. Since the dimensions of the grains are in the nanometer scale, each grain is considered as a single magnetic domain that reverses by uniform rotation. Thus, a unit vector $\sigma_{i}$ represents the magnetization orientation of each $\mathrm{F}$ grain, each $\mathrm{SG}$, each $\mathrm{SG}_{\mathrm{E}}$, and the interfacial uncompensated magnetization orientation of each bulk AF grain. At the interface, each $\mathrm{F}$ grain is coupled with an $\mathrm{AF}$ grain $\left(J_{\mathrm{F}-\mathrm{AF}}\right)$, or an $\mathrm{SG}$, or-in the case of nanodots-an $\mathrm{SG}_{\mathrm{E}}\left(J_{\mathrm{F}-\mathrm{SG}}\right)$ [Fig. 1(b)]. In agreement with the literature, the AF grains are decoupled from each other [25-27], as well as from the $S G$ and $S_{E}$ grains. In contrast, the $\mathrm{F}$ grains are coupled to each other $\left(J_{\mathrm{F}}\right)$ [Fig. 1(b)]. Uniaxial anisotropy along a common easy axis ( $y$ axis) in the plane of the layer is considered for all grains, and a linear thermal dependence of the anisotropy constants per unit volume $K_{i}$ is implemented [28]. Thus, the total energy of the system can be expressed as

$$
\begin{aligned}
E= & -\sum_{\langle i, j\rangle} J_{i j} \boldsymbol{\sigma}_{i} \cdot \boldsymbol{\sigma}_{j}-\sum_{i} K_{i} V_{i}\left(\boldsymbol{\sigma}_{i} \cdot \boldsymbol{e}_{y}\right)^{2} \\
& -\mu_{0} \mathbf{H} \cdot \sum_{i \in \mathrm{F}, \mathrm{SG}, \mathrm{SG}} \mathbf{m}_{\mathrm{E}},
\end{aligned}
$$

where $V_{i}$ is the grain volume, $\mathbf{m}_{i}$ is the magnetic moment of a $\mathrm{F}$ grain or a $\mathrm{SG}$ or a $\mathrm{SG}_{\mathrm{E}}$ (in the nanodots), and $\mathbf{H}$ is the magnetic field applied along the $y$ axis.

Most parameters of our model can be either found in literature or reasonably estimated from experimental data. The effective interfacial coupling per unit area, $j_{\mathrm{F}-\mathrm{AF}}$, can be deduced from the maximum value of $H_{E}$ in the case of an ideal $\mathrm{F} / \mathrm{AF}$ interface, i.e., without $\mathrm{SG}$, nor $\mathrm{SG}_{\mathrm{E}}$. Taking into account 
TABLE I. Fixed parameters of all our simulations.

\begin{tabular}{cccccc}
\hline \hline$j_{\mathrm{F}-\mathrm{AF}}\left(\mathrm{Jm}^{-2}\right)$ & $T_{N}(\mathrm{~K})$ & $K_{\mathrm{AF}}^{0}\left(\mathrm{Jm}^{-3}\right)$ & $K_{\mathrm{SG}}^{0}\left(\mathrm{Jm}^{-3}\right)$ & $t_{\mathrm{SG}}(\mathrm{nm})$ & $x_{\mathrm{SG}}(\%)$ \\
\hline $1.5 \times 10^{-4}$ & 690 & $4 \times 10^{5}$ & $3 \times 10^{5}$ & 2 & 50 \\
\hline \hline
\end{tabular}

the maximum value of $\left|H_{E}\right|$ measured in Ref. [10] for $t_{\mathrm{IrMn}}=$ $9 \mathrm{~nm}$, we found that $j_{\mathrm{F}-\mathrm{AF}}=1.5 \times 10^{-4} \mathrm{Jm}^{-2}$ is a reasonable value for $\mathrm{NiFe} / \mathrm{IrMn}$ bilayers. The $0-\mathrm{K}$ anisotropy constant for $\mathrm{AF}$ grains is taken to be $K_{\mathrm{AF}}^{0}=4 \times 10^{5} \mathrm{Jm}^{-3}$ [29]. The Néel temperature of the AF layer used in the expression of its anisotropy constant [28,30] is $T_{N}=690 \mathrm{~K}$ [3]. By contrast, the coupling per unit area, $j_{\mathrm{F}-\mathrm{SG}}$, and the effective anisotropy of $\mathrm{SG}$ and $\mathrm{SG}_{\mathrm{E}}$ are unknown. We assume that $j_{\mathrm{F}-\mathrm{SG}}$ is smaller or equal to $j_{\mathrm{F}-\mathrm{AF}}$. Similarly the effective anisotropy of SG and $\mathrm{SG}_{\mathrm{E}}$ is assumed to be weaker than that of the AF grains to report on the lower blocking temperatures of the disordered interfacial and dot edge phases demonstrated experimentally [16]. From a theoretical point of view, this can be justified because the noncollinearity of the spins in these phases should induce a decrease of the effective anisotropy constant. We arbitrarily set $K_{\mathrm{SG}}^{0}=0.75 K_{\mathrm{AF}}^{0}$. We point out that when $K_{\mathrm{SG}}^{0}$ increases, the blocking temperatures of $\mathrm{SG}$ and $\mathrm{SG}_{\mathrm{E}}$ increase which results in a shift toward larger temperatures of the low temperature part of the $H_{E}$ vs $T_{\mathrm{a}}$ (see Sec. III B 1) or $T_{\mathrm{M}}$ (see Sec. III B 2) curves. The fixed parameters of all our simulations are summarized in Table I. Note that the other parameters $j_{\mathrm{F}}, j_{\mathrm{F}-\mathrm{SG}}, t_{\mathrm{F}}$, and $t_{\mathrm{AF}}$ are given in each section.

Based on experimental measurements [31,32], disordered interfacial phases extend over 3-4 atomic planes, thus we set $t_{\mathrm{SG}}=2 \mathrm{~nm}$. In fact, it was demonstrated experimentally that the fraction of SG $x_{\mathrm{SG}}$ may be varied between about $20 \%$ and $80 \%$, as it is sensitive to interfaces and concomitant layers intermixing, and that it therefore depends on the stack and fabrication process [33]. For that in our simulations, we fixed the fraction of SG $x_{\mathrm{SG}}$ at the interface to an average value of $50 \%$ in both nanodots and continuous films. Let us mention that an increase of $x_{\mathrm{SG}}$ results in a small decrease of the exchange field at the temperatures above the maximum blocking temperature of the SG. Note that, in the case of nanodots, there is an additional contribution of grains with altered magnetic properties due to $\mathrm{SG}_{\mathrm{E}}$ (the fraction of $\mathrm{SG}$ and $\mathrm{SG}_{\mathrm{E}}$ is $58 \%$ on average for $L=130 \mathrm{~nm}$ ).

The simulations are performed in such a way as to reproduce experimental procedures [10,11]. First, in order to study the variability between nanodots in Sec. III A and to compare $H_{E}$ in nanodots with that in continuous films in Sec. III B 1, we simulate by the kinetic Monte Carlo method [34,35] the Soeya protocol [36]. This protocol consists of a first initial field cooling (FC) under a positive field $H_{\mathrm{FC}}$ from $T_{0}$ down to $T_{\mathrm{M}}=4 \mathrm{~K}$ (or $298 \mathrm{~K}$ ). Then successive annealings up to higher temperatures $T_{\mathrm{a}}$ such that $T_{\mathrm{M}} \leqslant T_{\mathrm{a}}<T_{0}$ followed by a FC under a negative field down to $T_{\mathrm{M}}$ are performed. This step is realized to gradually reorient the entities in contact with the $\mathrm{F}$ layer which satisfy $T_{\mathrm{B}}<T_{\mathrm{a}}$. Hysteresis loops are collected at $T_{\mathrm{M}}$ after each increment of $T_{\mathrm{a}}$. Thus, $T_{\mathrm{a}}$ is the variable parameter during the process. Second, to study size effect in Sec. III B 2, we use another procedure which consists of a unique $\mathrm{FC}$ under a positive field $H_{\mathrm{FC}}$ from $T_{0}$
TABLE II. Summary of parameter values used in Sec. III A.

\begin{tabular}{lc}
\hline \hline$t_{\mathrm{F}}=12 \mathrm{~nm}$ & $t_{\mathrm{AF}}=5 \mathrm{~nm}$ \\
$4 \times 10^{-5} \leqslant j_{\mathrm{F}}\left(\mathrm{Jm}^{-2}\right) \leqslant 4 \times 10^{-4}$ & $j_{\mathrm{F}-\mathrm{SG}}=8.5 \times 10^{-5} \mathrm{Jm}^{-2}$ \\
$T_{\mathrm{M}}=4 \mathrm{~K}$ (or $\left.298 \mathrm{~K}\right)$ & $T_{0}=550 \mathrm{~K}$ \\
\hline \hline
\end{tabular}

down to $5 \mathrm{~K}$. Then, successive hysteresis loops are measured at increasing temperatures $T_{\mathrm{M}}\left(T_{\mathrm{M}} \geqslant 5 \mathrm{~K}\right)$. Details of Monte Carlo simulations are given in Refs. [22,30].

It is worth noting that three regimes of grains in the AF layer in contact with the $\mathrm{F}$ layer should be differentiated according to their $T_{\mathrm{B}}$. Grains with $T_{\mathrm{B}}>T_{0}$ are not polarized by the field-cooling process; they remain randomly oriented with zero net magnetization and consequently do not contribute to $H_{E}$ in average. Grains with $T_{\mathrm{B}}<T_{\mathrm{M}}$ are polarized but are superparamagnetic at $T_{\mathrm{M}}$, thus their contribution to $H_{E}$ is null. Hence, only the grains with $T_{\mathrm{M}}<T_{\mathrm{B}}<T_{0}$ contribute to $H_{E}$.

\section{RESULTS AND DISCUSSIONS}

\section{A. Effect of $F$ grains coupling}

In this part, we investigate the effect of the effective coupling per unit area between the $\mathrm{F}$ grains $j_{\mathrm{F}}$ on $H_{E}$ measured at $T_{\mathrm{M}}$ versus the annealing temperature $\left(T_{\mathrm{a}}\right)$ for $\mathrm{NiFe}(12 \mathrm{~nm}) /$ IrMn $(5 \mathrm{~nm})$ nanodots and continuous films. To do so, we simulate the Soeya protocol mentioned in Sec. II from $T_{0}=$ $550 \mathrm{~K}$ down to $T_{\mathrm{M}}=4 \mathrm{~K}$ (or $298 \mathrm{~K}$ ). Within our simulations, the size of nanodots is $L=130 \mathrm{~nm}$, which corresponds to 289 grains per layer. The coupling between the $\mathrm{F}$ grains and $\mathrm{SG}$ is set to $j_{\mathrm{F}-\mathrm{SG}}=8.5 \times 10^{-5} \mathrm{Jm}^{-2}$. The parameter values are summarized in Table II.

Our results clearly show that there exist two different regimes depending on whether $j_{\mathrm{F}}$ is weak or strong compared to $2 K_{\mathrm{F}} l_{\mathrm{F}} / z_{\mathrm{F}}$ where $l_{\mathrm{F}}$ is the lateral dimension of the grain and $z_{\mathrm{F}}$ is the number of interacting $\mathrm{F}$ grains. As mentioned above, since $H_{E}$ is directly related to $T_{\mathrm{B}}$ of the grains in the AF layer (AF grains, $\mathrm{SG}$ and $\mathrm{SG}_{\mathrm{E}}$ in case of nanodots) in contact with the F layer, we plot the distribution of the intrinsic $T_{\mathrm{B}}\left(T_{\mathrm{B}}\right.$ in the absence of coupling and applied field) [23] of these entities in Fig. 2. We can see that since $T_{0}=550 \mathrm{~K}$ is larger than the maximum value of $T_{\mathrm{B}}$, all the grains in contact with the $\mathrm{F}$ layer are polarized during the initial $\mathrm{FC}$ and can contribute to $H_{E}$ depending on $T_{\mathrm{M}}$.

\section{Measurement temperature $T_{\mathrm{M}}=4 \mathrm{~K}$}

According to Fig. 2, all the grains which are in contact with the F layer are blocked at $T_{\mathrm{M}}=4 \mathrm{~K}$, so that all of these grains contribute to $H_{E}$. We first investigate the effect of $j_{\mathrm{F}}$ on the variability of $H_{E}$ from one nanodot to another. The $T_{\mathrm{a}}$ dependencies of $H_{E}$ for several nanodots with $j_{\mathrm{F}}=4 \times$ $10^{-4} \mathrm{Jm}^{-2}$ (strong coupling since $2 K_{\mathrm{F}} l_{\mathrm{F}} / z_{\mathrm{F}}=6.5 \times 10^{-5}$ for $z_{\mathrm{F}}=4$ ) and $4 \times 10^{-5} \mathrm{Jm}^{-2}$ (weak coupling) are plotted in Fig. 3. The variability is significant when $j_{\mathrm{F}}=4 \times 10^{-4} \mathrm{Jm}^{-2}$ whereas it decreases as $j_{\mathrm{F}}$ decreases (not shown) and vanishes for $j_{\mathrm{F}}=4 \times 10^{-5} \mathrm{Jm}^{-2}$. The $T_{\mathrm{a}}$ dependence of $H_{E}$ (averaged over 60 nanodots) is shown in Fig. 4 for $4 \times 10^{-5} \mathrm{Jm}^{-2}<$ $j_{\mathrm{F}}<4 \times 10^{-4} \mathrm{Jm}^{-2}$. We checked that averaging over a larger number of nanodots does not change the results. 

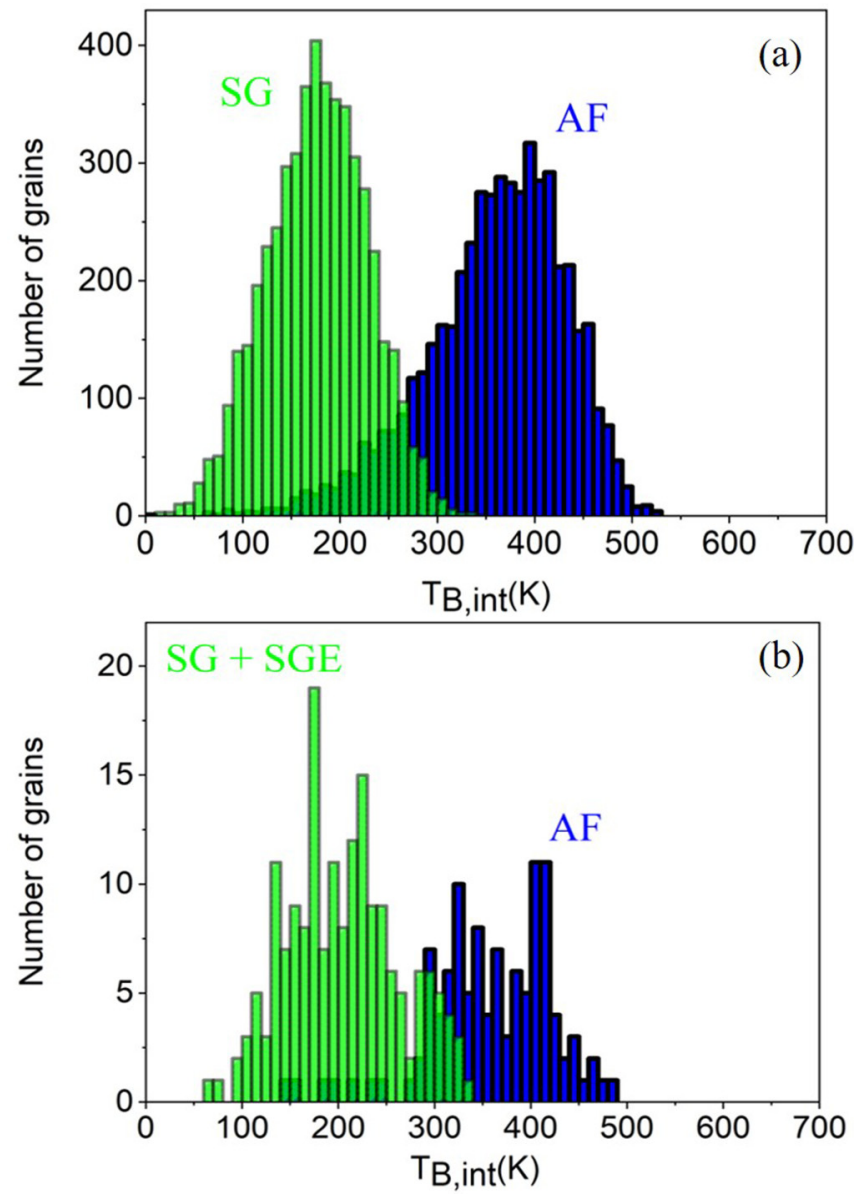

FIG. 2. Intrinsic blocking temperature distributions for the grains in the AF layer which are in contact with the $\mathrm{F}$ layer at the interface calculated from the volume distribution for (a) a continuous film (AF grains and SG) and (b) a nanodot with $L=130 \mathrm{~nm}$ (AF grains, SG, and $\mathrm{SG}_{\mathrm{E}}$ ) in $\mathrm{NiFe} / \operatorname{IrMn}(5 \mathrm{~nm})$ bilayers (the $\mathrm{AF}$ grains are in blue and the $\mathrm{SG}$ and $\mathrm{SG}_{\mathrm{E}}$ are in green).

We note that such an averaging is equivalent, experimentally, to make magneto-optical Kerr (MOKE) effect measurements on an array of nanodots with a laser spot size much larger than the pitch of the array $[9,11]$, or to perform magnetometry measurement on a full array. In contrast, data for isolated nanodots can be obtained experimentally either by MOKE when the size of the laser spot is smaller than the pitch of the array, or most likely through electrical measurements where each nanodot is contacted electrically, like in magnetic random access memories [21]. We compare our results with the expression of $H_{E}$ assuming that the $\mathrm{F}$ layer behaves as a macrospin coupled to the AF one with an average interfacial coupling $\left\langle j_{\text {int }}\right\rangle$ :

$$
H_{E}=\frac{\left\langle j_{\text {int }}\right\rangle}{\mu_{0} M_{\mathrm{F}} t_{\mathrm{F}}},
$$

where $M_{\mathrm{F}}$ is the magnetization of each $\mathrm{F}$ grain and $\left\langle j_{\text {int }}\right\rangle$ is expressed as

$$
\left\langle j_{\text {int }}\right\rangle=\frac{1}{2}\left[(1-2 r) j_{\mathrm{F}-\mathrm{AF}}+\left(1-2 r^{\prime}\right) j_{\mathrm{F}-\mathrm{SG}}\right],
$$

where $r$ is the fraction of AF grains in contact with the $\mathrm{F}$ layer negatively repolarized, and $r^{\prime}$ is the fraction of SG and
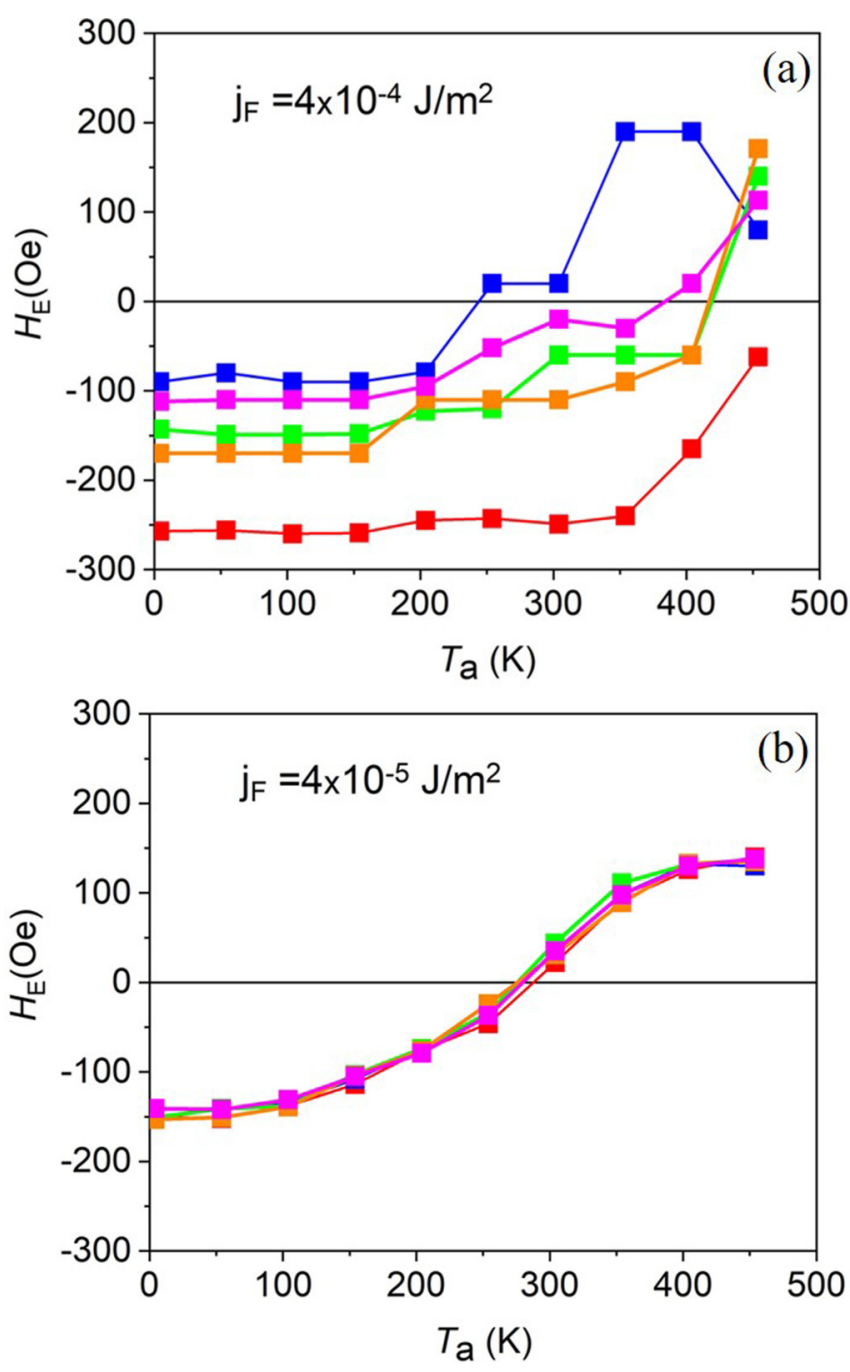

FIG. 3. $T_{\mathrm{a}}$ dependence of $H_{E}$ measured at $T_{\mathrm{M}}=4 \mathrm{~K}$ for (a) 5 $\mathrm{NiFe}(12 \mathrm{~nm}) / \operatorname{IrMn}(5 \mathrm{~nm})$ nanodots $(L=130 \mathrm{~nm})$ with $j_{\mathrm{F}}=4 \times$ $10^{-4} \mathrm{Jm}^{-2}$, and (b) $j_{\mathrm{F}}=4 \times 10^{-5} \mathrm{Jm}^{-2}$.

$\mathrm{SG}_{\mathrm{E}}$ negatively repolarized (these fractions increase with $T_{\mathrm{a}}$ ) [23]. It is important to note that within this assumption, $H_{E}$ does not depend on $j_{\mathrm{F}}$. From Fig. 4 , it can be seen that our simulated values of $H_{E}$ coincide with the average-coupling behavior given by Eq. (2) only if $j_{\mathrm{F}}$ is small enough (here $j_{\mathrm{F}}=$ $4 \times 10^{-5} \mathrm{Jm}^{-2}$ ). Thus our data clearly evidence two different regimes: an average-coupling behavior with no variability as $j_{\mathrm{F}}$ is weak enough and a $j_{\mathrm{F}}$ dependent behavior with high variability when $j_{\mathrm{F}}$ increases. It is also seen that the $j_{\mathrm{F}}$ dependence is more pronounced for higher $T_{\mathrm{a}}$. For a better understanding, the effect of $j_{\mathrm{F}}$ on the $\mathrm{F}$ layer reversal mechanism is shown in Fig. 5. We can see that for $j_{\mathrm{F}}=4 \times 10^{-4} \mathrm{Jm}^{-2}$, the F layer reversal starts with a grain located at one of the corners of the nanodot [red grain in Fig. 5(a)]. This grain acts as a nucleation center because it has a lower energy barrier due to a lack of $F$ neighbors and its small volume. Then due to the large value of $j_{\mathrm{F}}$, the magnetization reversal of the $\mathrm{F}$ layer propagates from this corner. So, the forward and backward reversal fields depend on the neighbors of the nucleation center $\left(\mathrm{AF}, \mathrm{SG}, \mathrm{SG}_{\mathrm{E}}\right.$ ) and on the number of $\mathrm{F}$ neighbors. 


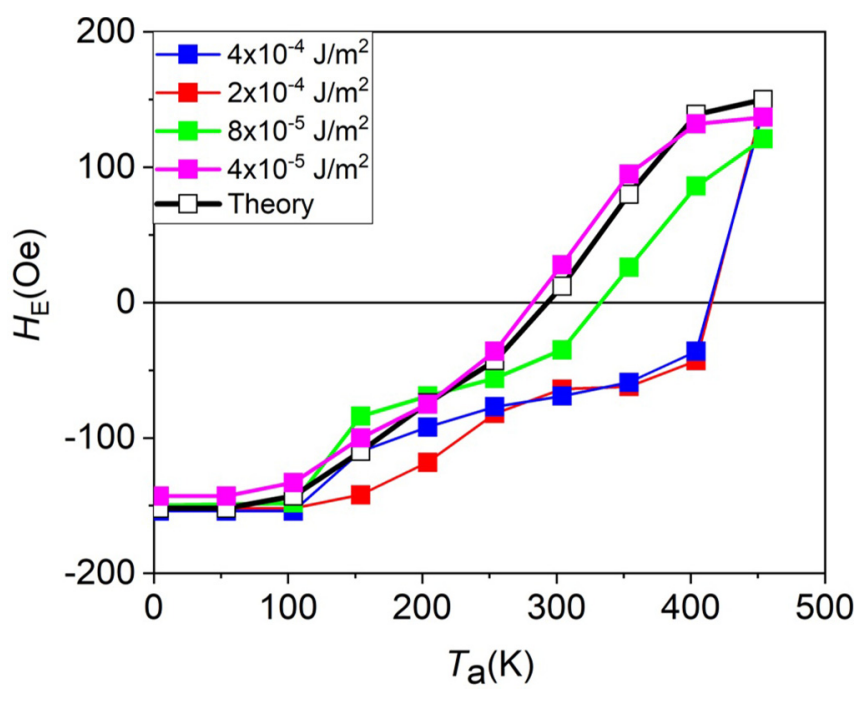

FIG. 4. $T_{\mathrm{a}}$ dependence of $H_{E}$ measured at $T_{\mathrm{M}}=4 \mathrm{~K}$ averaged over $60 \mathrm{NiFe}(12 \mathrm{~nm}) / \operatorname{IrMn}(5 \mathrm{~nm})$ nanodots $(L=130 \mathrm{~nm})$ for $4 \times$ $10^{-5} \mathrm{Jm}^{-2}<j_{\mathrm{F}}<4 \times 10^{-4} \mathrm{Jm}^{-2}$.

This environment differs between one nanodot and another leading to the observed variability in $H_{E}$. Such a variability actually agrees with earlier experimental results reported in [21]. On the contrary, for $j_{\mathrm{F}}=4 \times 10^{-5} \mathrm{Jm}^{-2}$, the reversal starts at different places [several nucleation centers, red grains of Fig. 5(b)] without propagation. Then F/AF coupling is averaged over these nucleation centers and $H_{E}$ is proportional to the average coupling $\left\langle j_{\text {int }}\right\rangle$. Thus the consequence is the absence of variability in that case which is desirable from a technological point of view. Finally, we can conclude that the average-coupling behavior (weak $j_{\mathrm{F}}$ ) is associated with a reversal with several nucleation centers without propagation, while the $j_{\mathrm{F}}$ dependent behavior (strong $j_{\mathrm{F}}$ ) corresponds to a reversal with a propagation from a single nucleation center.

\section{Measurement temperature $T_{\mathrm{M}}=298 \mathrm{~K}$}

For $T_{\mathrm{M}}=298 \mathrm{~K}$, only the grains in the AF layer which are in contact with the $\mathrm{F}$ layer at the interface, and having $298 \mathrm{~K}<T_{\mathrm{B}}<550 \mathrm{~K}$, contribute to $H_{E}$. From Fig. 2, we can see that a large fraction of AF grains contribute to $H_{E}$, whereas (a)

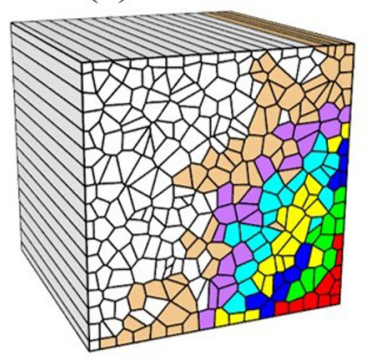

(b)

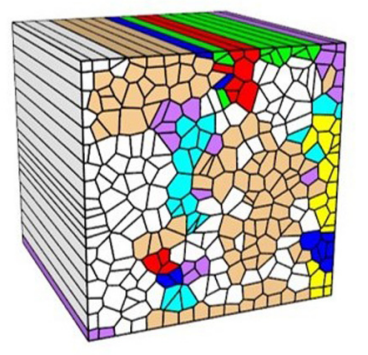

$1^{\text {st }} 11^{\text {th }} 21^{\text {th }} 31^{\text {th }} \quad 51^{\text {th }} \quad 71^{\text {th }} \quad 91^{\text {th }} \quad 160^{\text {th }} \quad$ grain reversal order

FIG. 5. F grain reversal sequence at $T_{\mathrm{M}}=4 \mathrm{~K}$ for (a) $j_{\mathrm{F}}=4 \times$ $10^{-4} \mathrm{Jm}^{-2}$ and (b) $j_{\mathrm{F}}=4 \times 10^{-5} \mathrm{Jm}^{-2}$ in $\mathrm{NiFe}(12 \mathrm{~nm}) / \operatorname{IrMn}(5 \mathrm{~nm})$ nanodots with $L=130 \mathrm{~nm}$.

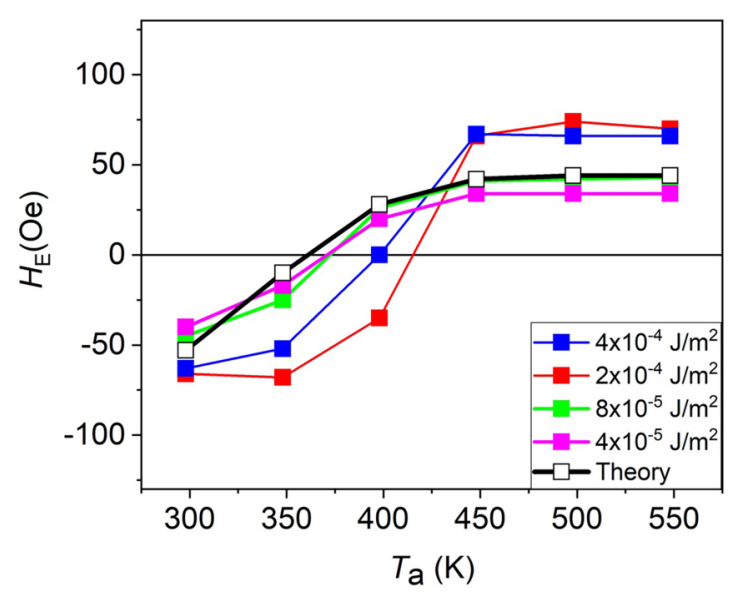

FIG. 6. $T_{\mathrm{a}}$ dependence of $H_{E}$ measured at $T_{\mathrm{M}}=298 \mathrm{~K}$ averaged over $60 \mathrm{NiFe}(12 \mathrm{~nm}) / \operatorname{IrMn}(5 \mathrm{~nm})$ nanodots $(L=130 \mathrm{~nm})$ for $4 \times$ $10^{-5} \mathrm{Jm}^{-2}<j_{\mathrm{F}}<4 \times 10^{-4} \mathrm{Jm}^{-2}$.

SG and $\mathrm{SG}_{\mathrm{E}}$ do not contribute. Similar to $T_{\mathrm{M}}=4 \mathrm{~K}$, we observe that the variability from one nanodot to another decreases as $j_{\mathrm{F}}$ decreases and vanishes for $j_{\mathrm{F}}=4 \times 10^{-5} \mathrm{Jm}^{-2}$ (not shown here). The $T_{\mathrm{a}}$ dependence of $H_{E}$ (averaged over 60 nanodots) for $4 \times 10^{-5} \mathrm{Jm}^{-2}<j_{\mathrm{F}}<4 \times 10^{-4} \mathrm{Jm}^{-2}$ is given in Fig. 6. Again, we compare our simulated values with the average-coupling behavior [Eq. (2)] where

$$
\left\langle j_{\text {int }}\right\rangle \approx \frac{(1-2 r)}{2} j_{\mathrm{F}-\mathrm{AF}} .
$$

As for $T_{\mathrm{M}}=4 \mathrm{~K}$, we find that the average-coupling behavior is satisfied if $j_{\mathrm{F}}$ is weak enough. Note that for $j_{\mathrm{F}}=$ $4 \times 10^{-4} \mathrm{Jm}^{-2}$, the $\mathrm{F}$ layer reversal does not start necessarily with a grain located at one corner of the nanodot unlike the case $T_{\mathrm{M}}=4 \mathrm{~K}$ due to thermal agitation (not shown here).

\section{B. Comparison with experimental data}

In this section, we investigate $\mathrm{NiFe} / \mathrm{IrMn}$ bilayers and we compare our results to experimental data $[10,11]$ in order to propose an explanation for the difference between the nanodot behavior and the continuous film behavior. Experimental findings [9,11] report MOKE measurements on arrays of nanodots with a laser spot size much larger than the pitch of the array. A spot of about 1-mm diameter for an array pitch of around $200 \mathrm{~nm}$ and a total array area of $1 \times 1 \mathrm{~mm}^{2}$ was used in Ref. [9], and complete $100 \times 100 \mu \mathrm{m}^{2}$ arrays of square dots with several lateral sizes 300 (interdot distance 100), 500 (interdot distance 300), and 1000 (interdot distance 300) nm were probed in Ref. [11]. As a consequence, the experimental data that were obtained correspond to an average over numerous nanodots. We therefore averaged our results over several nanodots where we found that 60 nanodots of averaging is enough for convergence. We took $j_{\mathrm{F}}=4 \times 10^{-5} \mathrm{Jm}^{-2}$ and $j_{\mathrm{F}-\mathrm{SG}}=1.5 \times 10^{-4} \mathrm{Jm}^{-2}$.

\section{NiFe/IrMn bilayers $\left(T_{\mathrm{M}}=298 \mathrm{~K}\right)$}

Here we investigate the $T_{\mathrm{a}}$ dependence of $H_{E}$ for various $t_{\mathrm{IrMn}}$ with $t_{\mathrm{NiFe}}=12 \mathrm{~nm}$. The simulation procedure is the same as in Sec. II (Soeya protocol) with an initial FC under 


\section{(a) Our simulations}

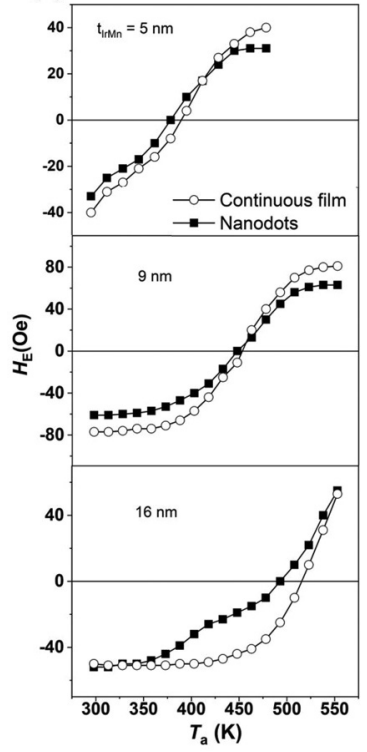

(b) Experimental data

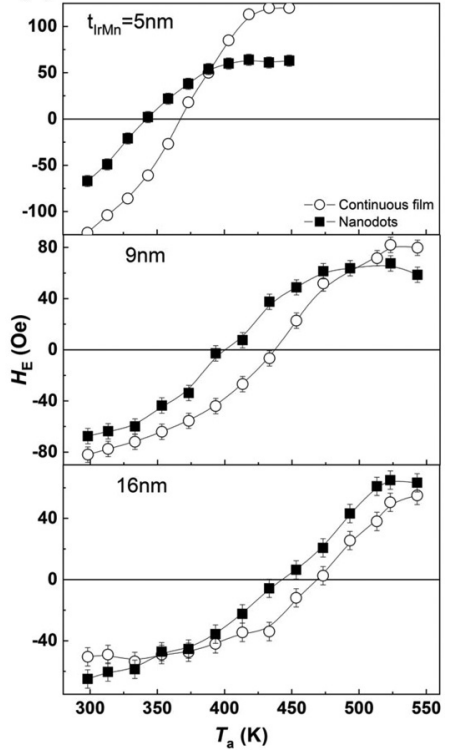

FIG. 7. Simulated $T_{\mathrm{a}}$ dependence of $H_{E}$ measured at $T_{\mathrm{M}}=298 \mathrm{~K}$ for (a) NiFe/IrMn bilayers $\left(t_{\mathrm{IrMn}}=5,9\right.$, and $\left.16 \mathrm{~nm}\right)$, compared to experimental data [10] (b).

$H_{\mathrm{FC}}=2.4 \mathrm{kOe}$ from $T_{0}=550 \mathrm{~K}$ down to $T_{\mathrm{M}}=298 \mathrm{~K}$. In Fig. 7(a), we show the simulated $T_{\mathrm{a}}$ dependence of $H_{E}$ for $t_{\mathrm{IrMn}}=5,9$ and $16 \mathrm{~nm}$. The parameter values are summarized in Table III. A shift of the $H_{E}$ curve to higher temperatures as $t_{\mathrm{IrMn}}$ increases is observed which is in good qualitative agreement with experimental observations [Fig. 7(b)] [10]. We can explain these results as follows: (i) Here, the main contribution to $H_{E}$ is due to the $\mathrm{AF}$ grains (those with $298 \mathrm{~K}<$ $T_{\mathrm{B}}<550 \mathrm{~K}$ ), the $\mathrm{SG}$ do not contribute, and the $\mathrm{SG}_{\mathrm{E}}$ give a small contribution only for the nanodot with $t_{\mathrm{IrMn}}=16 \mathrm{~nm}$; (ii) then the derivative $d H_{E} / d T_{\mathrm{a}}$ is proportional to the $T_{\mathrm{B}}$ distribution of the $\mathrm{AF}$ grains contributing to $H_{E}$ [22,23]; (iii) consequently, since the $T_{\mathrm{B}}$ distribution of the $\mathrm{AF}$ grains shifts to higher temperatures as $t_{\mathrm{IrMn}}$ increases the curve of the derivative and thus the $H_{E}$ curve versus $T_{\mathrm{a}}$ shifts towards higher value of $T_{\mathrm{a}}$ as $t_{\mathrm{IrMn}}$ increases. In agreement with experimental data, the simulated value of $\left|H_{E}\right|$ at $T_{\mathrm{a}}=298 \mathrm{~K}$ is smaller in nanodots for $t_{\mathrm{IrMn}}=5 \mathrm{~nm}$ and $9 \mathrm{~nm}$ which can be explained by the presence of $\mathrm{SG}_{\mathrm{E}}$ in nanodots which do not contribute to $H_{E}$ for these thicknesses. On the contrary, $\left|H_{E}\right|$ at $T_{\mathrm{a}}=298 \mathrm{~K}$ is slightly larger in nanodots for $t_{\mathrm{IrMn}}=16 \mathrm{~nm}$ in qualitative agreement with the experimental data. These results come from the contribution of $\mathrm{SG}_{\mathrm{E}}$ since their $T_{\mathrm{B}}$ (which increases with $t_{\mathrm{IrMn}}$ ) is in the window delimited by $550 \mathrm{~K}$ and $298 \mathrm{~K}$ for this AF thickness. We insist on the fact

TABLE III. Summary of parameter values used in Sec. III B 1.

\begin{tabular}{lc}
\hline \hline$t_{\mathrm{F}}=12 \mathrm{~nm}$ & $t_{\mathrm{AF}}=5,9$, and $16 \mathrm{~nm}$ \\
$j_{\mathrm{F}}=4 \times 10^{-5} \mathrm{Jm}^{-2}$ & $j_{\mathrm{F}-\mathrm{SG}}=1.5 \times 10^{-4} \mathrm{Jm}^{-2}$ \\
$T_{\mathrm{M}}=298 \mathrm{~K}$ & $T_{0}=550 \mathrm{~K}$ \\
\hline \hline
\end{tabular}

TABLE IV. Summary of parameter values used in III B 2.

\begin{tabular}{lc}
\hline \hline$t_{\mathrm{F}}=8 \mathrm{~nm}$ & $t_{\mathrm{AF}}=8 \mathrm{~nm}$ \\
$j_{\mathrm{F}}=4 \times 10^{-5} \mathrm{Jm}^{-2}$ & $j_{\mathrm{F}-\mathrm{SG}}=1.5 \times 10^{-4} \mathrm{Jm}^{-2}$ \\
$5 \leqslant T_{\mathrm{M}}(\mathrm{K}) \leqslant 300$ & $T_{0}=300 \mathrm{~K}$ \\
\hline \hline
\end{tabular}

that all the parameters except $t_{\mathrm{IrMn}}$ were kept the same for the six simulated curves given in Fig. 7(a).

\section{NiFe/IrMn bilayers $\left(T_{M}=\right.$ variable $)$}

Our aim here is to investigate the lateral size effects on the temperature dependence of $H_{E}$ in NiFe/IrMn systems with $t_{\mathrm{NiFe}}=t_{\mathrm{IrMn}}=8 \mathrm{~nm}$ in comparison with Ref. [11]. The procedure consists of a unique $\mathrm{FC}$ under $H_{\mathrm{FC}}=500 \mathrm{Oe}$ from $T_{0}=300 \mathrm{~K}$ down to $5 \mathrm{~K}$. Then, successive hysteresis loops are measured at increasing temperatures $T_{\mathrm{M}} \geqslant 5 \mathrm{~K}$. The parameter values are summarized in Table IV. The simulated temperature dependence of $\left|H_{E}\right|$ is shown in Fig. 8(a). Again, a good qualitative agreement with the experimental data in Fig. 8(b) is obtained: $\left|H_{E}\right|$ is larger in small nanodots at $5 \mathrm{~K}$ whereas it is the opposite as the temperature increases (for $T_{\mathrm{M}}$ around $150-200 \mathrm{~K}$ ). The reason is that at $T_{\mathrm{M}}=5 \mathrm{~K}$, in both systems, only a small fraction of AF grains (those with $5 \mathrm{~K}<$ $T_{\mathrm{B}}<300 \mathrm{~K}$ since $T_{0}=300 \mathrm{~K}$ ) and all SG contribute to $H_{E}$. But in the case of nanodots, there is an additional contribution due to $\mathrm{SG}_{\mathrm{E}}$. Consequently, $\left|H_{E}\right|$ is larger in nanodots. Since the fraction of $\mathrm{SG}_{\mathrm{E}}$ decreases as the size increases, $\left|H_{E}\right|$ decreases when the size increases. As $T_{\mathrm{M}}$ increases, the fraction of SG and $\mathrm{SG}_{\mathrm{E}}$ that contribute to $H_{E}$ decreases which explains the decrease in $\left|H_{E}\right|$. For $T_{\mathrm{M}}>150 \mathrm{~K},\left|H_{E}\right|$ becomes larger in continuous films because there are more SG polarized towards the positive orientation of the field, thus the positive reversal field becomes smaller in continuous films. Note that we observe a difference between the simulated values of $\left|H_{E}\right|$ and the experimental ones because we choose the value of $j_{\mathrm{F}-\mathrm{AF}}$ to fit the maximum value of $\left|H_{E}\right|$ for $t_{\mathrm{IrMn}}=9 \mathrm{~nm}$ of Ref. [10]. However, the present experimental data have been obtained in two different laboratories on distinct samples. So the quality of the F/AF interface is likely not the same due to the differences in the fabrication process, i.e., the effective interfacial F-AF coupling in the present samples is larger.
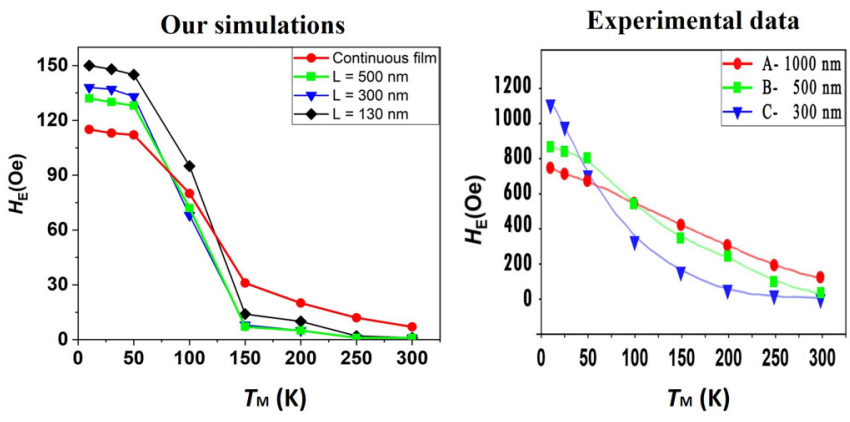

FIG. 8. Simulated temperature dependence of $\left|H_{E}\right|$ for $\mathrm{NiFe} / \mathrm{IrMn}$ bilayers $\left(t_{\mathrm{IrMn}}=8 \mathrm{~nm}\right)$ for (a) various nanodot sizes and continuous film in comparison with experimental data [11] (b). 


\section{CONCLUSIONS}

In this paper, we investigated the effects of reducing lateral sizes in F/AF nanodots using a granular model and kinetic Monte Carlo simulations. Our granular model took into account less stable magnetic grains at the F/AF interface due to atomic diffusions, stacking faults, etc. In the case of nanodots, additional less stable grains at the edges in the AF layer, due to the nanofabrication are considered. Our results demonstrated the crucial impact of the coupling between $\mathrm{F}$ grains. We evidenced that two different mechanisms of the $\mathrm{F}$ layer reversal can occur. An average-coupling behavior occurs with no variability from one nanodot to another when $j_{\mathrm{F}}$ is weak enough, corresponding to a reversal mechanism relying on several nucleation centers at any location within the film. In contrast, a $j_{\mathrm{F}}$ dependent behavior with unwanted [21] high variability from one nanodot to another takes place when $j_{\mathrm{F}}$ increases, corresponding to a reversal mechanism highly dependent on a single nucleation center at one corner of the nanodot and subsequent domain wall propagation. Our model also robustly accounts for several features experimentally observed in exchange-biased nanodots after several nontrivial experimental procedures are used to set the EB field by using only a single set of parameters.

\section{ACKNOWLEDGMENTS}

This project is funded by the Région Normandie and the European Union. Europe invests in Normandy with the European Regional Developement Fund (ERDF) - MAGMA project. The authors acknowledge the Centre Régional Informatique et d'Applications Numériques de Normandie (CRIANN) where simulations were performed as Project No. 2010006. We also thank Ahmed Albaalbaky for critical reading of the manuscript.
[1] W. H. Meiklejohn and C. P. Bean, Phys. Rev. 102, 1413 (1956).

[2] W. H. Meiklejohn and C. P. Bean, Phys. Rev. 105, 904 (1957).

[3] J. Noguès and I. K. Schuller, J. Magn. Magn. Mater. 192, 203 (1999).

[4] A. E. Berkowitz and K. Takano, J. Magn. Magn. Mater. 200, 552 (1999).

[5] I. L. Prejbeanu, M. Kerekes, R. C. Sousa, H. Sibuet, O. Redon, B. Dieny, and J. P. Nozieres, J. Phys.: Condens. Matter 19, 165218 (2007).

[6] V. Baltz, A. Manchon, M. Tsoi, T. Moriyama, T. Ono, and Y. Tserkovnyak, Rev. Mod. Phys. 90, 015005 (2018).

[7] C. Chappert, A. Fert, and F. N. Van Dau, Nat. Mater. 6, 813 (2007).

[8] J. Noguès, J. Sort, V. Langlais, V. Skumryev, S. Surinach, J. S. Munoz, M. D. Baro, and I. K. Schuller, Phys. Rep. 422, 65 (2005).

[9] V. Baltz, J. Sort, S. Landis, B. Rodmacq, and B. Dieny, Phys. Rev. Lett. 94, 117201 (2005).

[10] V. Baltz, J. Sort, B. Rodmacq, B. Dieny, and S. Landis, Phys. Rev. B 72, 104419 (2005).

[11] F. Spizzo, E. Bonfiglioli, M. Tamisari, A. Gerardino, G. Barucca, A. Notargiacomo, F. Chinni, and L. Del Bianco, Phys. Rev. B 91, 064410 (2015).

[12] I. Sasaki, R. Nakatani, K. Ishimoto, Y. Endo, Y. Shiratsuchi, Y. Kawamura, and M. Yamamoto, J. Magn. Magn. Mater. 310, 2677 (2007).

[13] Y. Shen, Y. Wu, H. Xie, K. Li, J. Qiu, and Z. Guo, J. Appl. Phys. 91, 8001 (2002).

[14] G. Vinai, G. Gaudin, J. Moritz, J. Vogel, I. L. Prejbeanu, and B. Dieny, J. Phys. D: Appl. Phys. 47, 195302 (2014).

[15] S. Laureti, S. Y. Suck, H. Haas, E. Prestat, O. Bourgeois, and D. Givord, Phys. Rev. Lett. 108, 077205 (2012).

[16] V. Baltz, G. Gaudin, P. Somani, and B. Dieny, Appl. Phys. Lett. 96, 262505 (2010).

[17] G. Garcia, M. Kiwi, J. Mejia-Lopez, and R. Ramirez, J. Magn. Magn. Mater. 322, 3329 (2010).

[18] G. Vallejo-Fernandez and J. N. Chapmam, Appl. Phys. Lett. 94, 262508 (2009).
[19] A. P. Malozemoff, Phys. Rev. B 35, 3679 (1987).

[20] M. P. Proenca, J. Ventura, C. T. Sousa, M. Vazquez, and J. P. Araujo, Phys. Rev. B 87, 134404 (2013).

[21] K. Akmaldinov, L. Frangou, C. Ducruet, C. Portemont, J. Pereira, I. Joumard, B. Dieny, J. Alvarez-Hérault, and V. Baltz, IEEE Magn. Lett. 6, 3000404 (2015).

[22] G. Lhoutellier, D. Ledue, R. Patte, F. Barbe, B. Dieny, and V. Baltz, J. Phys. D: Appl. Phys. 48, 115001 (2015).

[23] G. Lhoutellier, D. Ledue, R. Patte, and V. Baltz, J. Appl. Phys. 120, 193902 (2016).

[24] R. Quey, P. R. Dawson, and F. Barbe, Comput. Methods Appl. Mech. Eng. 200, 1729 (2011).

[25] E. Fulcomer and S. H. Charap, J. Appl. Phys. 43, 4190 (1972).

[26] D. Choo, R. W. Chantrell, R. Lamberton, A. Johnston, and K. O'Grady, J. Appl. Phys. 101, 09E521 (2007).

[27] B. Craig, R. Lamberton, A. Johnston, U. Nowak, R. W. Chantrell, and K. O'Grady, J. Appl. Phys. 103, 07C102 (2008).

[28] M. D. Stiles and R. D. McMichael, Phys. Rev. B 60, 12950 (1999).

[29] G. Vallejo-Fernandez, L. E. Fernandez-Outon, and K. O'Grady, Appl. Phys. Lett. 91, 212503 (2007).

[30] D. Ledue, A. Maitre, F. Barbe, and L. Lechevallier, J. Magn. Magn. Mater. 372, 134 (2014).

[31] L. Lechevallier, A. Zarefy, R. Lardé, H. Chiron, J. M. Le Breton, V. Baltz, B. Rodmacq, and B. Dieny, Phys. Rev. B 79, 174434 (2009).

[32] L. Lechevallier, A. Zarefy, F. Letellier, R. Lardé, D. Blavette, J. M. Le Breton, V. Baltz, B. Rodmacq, and B. Dieny, J. Appl. Phys. 112, 043904 (2012).

[33] K. Akmaldinov, S. Auret, I. Dieny, and V. Baltz Appl. Phys. Lett. 103, 042415 (2013).

[34] D. W. Heermann, Computer Simulation Methods in Theoretical Physics, 2nd ed. (Springer, Berlin, 1990).

[35] K. Binder and D. W. Heermann, Monte Carlo Simulation in Statistical Physics, 2nd ed. (Springer, Berlin, 1990).

[36] S. Soeya, T. Imagawa, K. Mitsuoka, and S. Narishige, J. Appl. Phys. 76, 5356 (1994). 